\title{
THE QUANTITATIVE PROBLEM FOR THEORIES OF DYSFUNCTION AND DISEASE
}

\author{
Thomas Schramme ${ }^{1}$ \\ ${ }^{1}$ University of Liverpool \\ Original scientific article - Received: 22/03/2021 Accepted: 08/08/2021
}

\begin{abstract}
Many biological functions allow for grades. For example, secretion of a specific hormone in an organism can be on a higher or lower level, compared to the same organism at another occasion or compared to other organisms. What levels of functioning constitute instances of dysfunction; where should we draw the line? This is the quantitative problem for theories of dysfunction and disease. I aim to defend a version of biological theories of dysfunction to tackle this problem. However, I will also allow evaluative considerations to enter into a theory of disease. My argument is based on a distinction between a biological and a clinical perspective. Disease, according to my reasoning, is restricted to instances that fall within the boundaries of biological dysfunctions. Responding to the quantitative problem does not require arbitrary decisions or social value-judgements. Hence, I argue for a non-arbitrary, fact-based method to address the quantitative problem. Still, not all biological dysfunctions are instances of disease. Adding a clinical perspective allows us to prevent the potential over-inclusiveness of the biological perspective, because it restricts the boundaries of disease even further.
\end{abstract}

Keywords: theory of function; dysfunction; line-drawing problem; concept of disease; nosology 


\section{Introduction}

Many biological functions allow for grades. For example, secretion of a specific hormone in an organism can be on a higher or lower level, compared to the same organism at another occasion or compared to other organisms. What levels of functioning constitute instances of dysfunction; where should we draw the line? This is the quantitative problem for theories of dysfunction and disease. It has increasingly been discussed in the philosophy of medicine in the past few years (Schwartz 2007; Hausman 2014; Griffiths and Matthewson 2016; Rogers and Walker 2017). Partly, the discussion is connected to the established debate between naturalism and normativism about the concept of disease. It seems that drawing boundaries between grades of normal and abnormal functioning involves value judgements, which undermine the naturalist ambition to devise a value-free theory of disease. In addition, the lack of a clear and widely accepted procedure for drawing the line seems to allow pathologisation of normal conditions as well as overdiagnosis (cf. Schramme 2019, 91ff.; Hofmann 2021). Every level of somewhat low organismic functioning seems to constitute a potential disease, if the line can only be drawn on the basis of human interests.

These practical concerns will form the backdrop of my contribution to the recent philosophical debate. I aim to defend a version of biological theories of dysfunction that exclude social value judgements. However, I will also allow evaluative considerations based on human interests to enter into theories of disease. My argument is based on a distinction between a biological and a clinical perspective (cf. Boorse 2014; Tresker 2020). The concept of disease, according to my reasoning, should be restricted to instances of biological dysfunctions. The use of 'should', in this context, implies that I do not believe in the possibility of pure conceptual analysis, resulting in a real definition of disease (cf. Lemoine 2013; Varga 2018). The best theory of disease will be determined by scientific considerations in combination with pragmatic interests, such as the avoidance of overdiagnosis.

Responding to the quantitative problem does not require arbitrary decisions or social value-judgements. Hence, I argue for a non-arbitrary, fact-based method to draw the boundary of dysfunction. Still, not all biological dysfunctions are instances of disease. Adding a clinical perspective allows us to prevent the potential over-inclusiveness of the biological perspective-in terms of potentially including too many diseases if we identify disease with biological dysfunction. To add a 
clinical perspective helps to restrict the boundaries of disease to medically relevant dysfunctions.

In section 1 , I introduce the quantitative problem within the context of a theory regarding the absolute concept of disease, that is, a conception that does not allow for grades of diseasedness. Section 2 briefly discusses the qualitative problem-which is concerned with identifying functions as opposed to non-functional mechanisms - in order to better understand the main concern of this paper. Only mechanisms that are identified as proper, performing functions are relevant for a theory of function and, derivatively, for a theory of disease. Hence, only functional traits are relevant for the quantitative problem. Section 3 then more thoroughly looks at the quantitative problem, specifically at Christopher Boorse's attempt to address it. I argue that this attempt struggles as it is, but can be repaired by adding clarity about the implications of seeing functions as effects within an organismic system. Thresholds for sufficient levels of functioning are determined in relation to next-level functions and the overall maintenance of the system. Accordingly, effectiveness of functioning is the relevant criterion for answering the quantitative problem, not the functional efficiency of a trait. In section 4, I draw a closer connection to medicine by introducing a perspective of clinical dysfunction, which is a narrower category than biological dysfunction. In section 5, I discuss the application of the general classification of clinical dysfunctions, which can be found in nosological systems, to individual patients through the process of medical diagnosis. Diagnosis therefore involves some discretion for clinicians when determining the boundary between normal functioning and pathology in individual cases. However, this practice is only possible within the boundaries set by the scientific notion of biological dysfunction. It therefore does not introduce wholly arbitrary elements. Section 6 concludes.

\section{The Quantitative Problem in the Context of an Absolute Concept of Disease}

In medicine, it is usually said that disease is the absence of health (in a specific respect, say, in respect to one's respiratory system). Health is deemed the opposite of disease. It is true that this conceptual binarity by itself does not establish clear-cut boundaries. Still, when we talk in this way, we interpret disease as an absolute concept. There are no grey areas; conditions either constitute a state of health or of disease. Things might be different when we consider whether a person is healthy, that is, when we consider health from a holistic perspective. From such a perspective, we 
can easily consider conditions of disease that are consistent with a person being overall healthy.

From an absolute conceptual framework, we can also allow for positive health to be a gradable notion. We might consider healthier-than judgements, where one person is compared to another person (Schroeder 2013); yet these judgements do not result in grades of disease, because being less healthy is not the same as being unhealthy or more diseased. Disease is, so to speak, below the threshold of minimal health. It is true, of course, that different instantiations of diseases pose different levels of severity. Accordingly, we might want to say that a particular disease is more clearly a case of disease than another. Yet, if we have determined whether a condition is a disease, then it simply belongs to the class of disease, never mind how serious it is.

Such an absolute perspective is quite important in many practical contexts, most significantly when the presence of disease is used as a kind of entry ticket to the system of publicly funded medical resources. Here we need an absolute statement as to whether a condition is justifiably deemed a disease or not. If a condition is not a disease, it ought not to be treated by using publicly funded resources, at least not without additional argument. A condition that constitutes a disease, on the other hand, is a legitimate concern of a public health system without further reasons; although this might still not be enough to guarantee the public funding of treatment under the usual conditions of scarcity.

A serious problem for medical theory with respect to establishing an absolute concept of disease that has recently gained momentum is where exactly to draw the threshold between health and disease. What criteria need to be fulfilled in order to classify a condition as a disease? A common way to draw this boundary is to establish the criterion of dysfunction, or more exactly of impairment of functional ability (Boorse 1977). For the purposes of this paper, I will take such a Boorsean framework for granted, though I divert from Boorse in several respects. Accordingly, the general concept of disease is understood as impairment of functional ability. Functional ability is the readiness of a trait, for instance an organ, to 'pursue' its tasks. Accordingly, a trait currently not doing any work is still functional, perhaps due to environmental causes (Garson 2019, 126ff), if it has the relevant functional ability. Disease can therefore be understood as impairments of relevant dispositions within the Boorsean theoretical framework (Boorse 2014, 685). I will later identify disease with clinical dysfunction, which is based on, but not identical to, biological dysfunction. 
Such a distinction is not thoroughly discussed in Boorse's theory, yet he explicitly allows for a clinical perspective on disease (Boorse 1997, 48).

There is an important difference between the theoretical problem of delineating disease as opposed to the same problem posed from a practical point of view. A doctor who deals with a suffering patient is not primarily interested in whether the organism in front of her is dysfunctional, but in her patient's wellbeing, broadly conceived. The doctor might therefore be tempted to identify a disease where there is no dysfunction or, conversely, not to diagnose a condition in terms of disease despite its being dysfunctional. It is important to disentangle different contexts of referring to dysfunction and disease, because they are based on different types of interests. I will distinguish between two such contexts: A biological and a clinical context.

From a theoretical point of view, aiming at an explanation of the concept of disease, the focus on the notion of dysfunction as a necessary criterion of disease allows us to establish an absolute concept of disease. Only where there is dysfunction, there can be disease. We are accordingly pushed back to the level of organismic functions and their impairments. However, individual organismic conditions and processes come in degrees. For instance, secretion of hormones allows for different values in different organisms at different times and under different environmental circumstances. Accordingly, when we focus on dysfunction as the basis of the concept of disease we seem to enter a grey area, after all, because the exact level of function that allows for a process to be called $d y s f u n c t i o n a l$ appears to be insurmountably vague. In other words, whether the concept of dysfunction allows for absolute thresholds and whether these can be established scientifically is not straightforward.

Some levels of performance can be deemed unambiguously dysfunctional, simply because they completely lack in functioning. Since a function is an effect of a trait, if a trait does not produce any such effect, it is dysfunctional. For instance, if a heart does not pump blood at all, it is dysfunctional. But surely there are many instances of organismic mechanisms producing effects that are however not sufficient to be deemed functioning. The problem discussed in this paper is how and where to draw this very line. I call it the quantitative problem of theories of dysfunction, because it is concerned with the level of producing an effect, not with the kind of effect a function is supposed to achieve.

Other authors have called the problem I will address "the line drawing problem", most notably Peter Schwartz (2007), who was one of the first 
authors to bring it up explicitly under this label in the philosophy of medicine, although there are important precursors to the recent debate (Engelhardt 1976; Goosens 1980). Boorse $(1977,1987,1997)$ did attempt to tackle this problem in the past, from a naturalist point of view, but I believe there are problems with his account. Only quantifiable functions raise serious concerns where to draw the line; lack of any functional effect straightforwardly constitutes dysfunction. That is why I think the line drawing problem, which generally asks for the line between the functional and the dysfunctional, is in reality restricted to the quantitative problem. Accordingly, I prefer the latter label.

The term 'problem' is slightly ambiguous and it might be helpful to briefly explain in what sense I intend to tackle the quantitative problem. First, a problem can be something that is generally a matter of concern, for instance, especially in our context, a philosophical problem. In this way, a philosophical problem might never be solved; it might continue to be a matter of interest or concern, something that requires explaining. The mind-body problem might be a fitting illustration. It might never be solved and continues to interest us from a philosophical point of view. Second, a problem can also be something that bothers us in a certain way or that we want to get rid of. The mind-body problem might not be a problem in this second sense. Now, I believe the quantitative problem will continue to be a problem in the first sense of the term. It will continue to raise philosophical concerns. In this paper, I want to show a way to address the quantitative problem in a way that eases the problem in the second sense of the term, especially by showing a reasonable, non-arbitrary and workable way to conceptualise dysfunction and disease. I will not solve the problem in the first sense and will therefore avoid speaking of a solution to avoid any confusion.

In this way, I will defend an answer to the quantitative problem which claims to rely only on scientific aspects, hence avoids external evaluative elements, for instance in relation to individual harm, as other authors have introduced. The main idea is to identify the relevant level of gradable functioning with achieving a particular effect, relative to other functions of an organism. It is argued that the relevant threshold of quantitative functioning is determined by the biological necessities that are involved when a part of an organism, understood as an overarching system, is to perform its biological functions. Functions are effects, and any such effect is a means to maintain other functions, altogether maintaining the system as a whole. We can determine the required level of functioning in relation to the structured sub-systems of an organism. The quantitative problem therefore raises scientific questions regarding the biological organisation 
of organisms. However, I will also argue that this only relates to the biological perspective. I suggest that in medicine we further need to account for a clinical perspective regarding the boundary between normal functioning and dysfunction. The clinical perspective introduces additional features, which are partly evaluative and pragmatic.

\section{The Qualitative Problem}

We can contrast the quantitative problem of dysfunction with the qualitative problem. The qualitative problem is concerned with identifying the kinds of traits of organisms that can be deemed functional, as opposed to being non-functional. Note that 'non-functional' means 'having no function'; it does not mean 'dysfunctional'. For instance, the function of the heart is to pump blood, not to produce noise, though the latter is also an effect of the organ's mechanisms. So, in other words, the qualitative problem aims at identifying the functions of traits. In the philosophy of biology, and also in the philosophy of medicine, this has been the major concern in the last decades. Several theories have been offered as to how to account for functions (a good range of papers can be found in Buller 1999 and Ariew et al. 2002; see also Garson 20016, for a helpful overview). I will not discuss these theories, because my main focus is on the quantitative problem.

To be sure, I do not want to deny that there is a close connection between the qualitative and the quantitative problem. After all, identifying functions (i.e. tackling the qualitative problem) usually comes with specific quantitative levels of functioning (see Schwartz 2007, 366). So, for instance, the heart does not simply have the function to pump blood but to pump about 5 litres per minute in a resting adult person. I still want to insist on the difference between the two problems for analytic purposes, because later I will argue that examples of quantifiable non-functional traits do not apply to the quantitative problems. In other words, only functional traits raise the relevant problem. In general, it seems to me that the second aspect of the qualitative problem-specifying functions over and above identifying traits with functions - can be translated into the quantitative problem, because it causes the need to determine thresholds for normal functioning.

Many theories of function can account for dysfunction or malfunction. This is usually, though not universally, done by using the type-token distinction (Godfrey-Smith 1993, 200). More specifically, types of traits are explained to have specific functions. In the medical context, 'trait' may 
stand for organismic sub-systems, such as the respiratory system, organs, cells, or even genes. All of these things may have a function, and it is of course an important problem for biological and medical research to explore these functions. Different theories of functions differ in their explanations as to why a particular effect is the function of a trait. It might be due to its evolutionary history (Wright 1973; Millikan 1989; Neander 1991), its contribution to a larger system's capacities (Cummins 1975), or to the good of the organism (Melander 1997; McLaughlin 2001; Wouters 2003). Once a type of a functional trait is established, tokens can be assessed according to the norm set by the functional type. Accordingly, the qualitative problem regarding dysfunction is concerned with identifying those features, or qualities, of organisms that can be dysfunctional at all. If a trait does not have a function, it cannot be dysfunctional.

The qualitative problem also addresses the problem of how a trait can be dysfunctional. Once the function of a trait is established, we know in what way a token can be dysfunctional, namely in terms of the effect that is its function, not by lacking in terms of other effects. For instance, a heart can be dysfunctional in terms of blood-pumping, not in terms of noiseproduction.

This is all I will say about the qualitative problem. It should nevertheless be pointed out that many issues in relation to the qualitative problem have not been sufficiently tackled in the philosophy of biology and the philosophy of medicine, for instance the related problem whether proper functions come in degrees (Matthewson 2020). Most notably, the specific normativity of function statements, which is supposed to account for the possibility of dysfunction, or malfunction, is also still a contested issue (Neander 1995; Davies 2001; Garson 2019).

\section{Taking the Sting Out of the Quantitative Problem}

As I have said already, the quantitative problem regarding dysfunction is due to the fact that many functions allow for degrees. At least in some contexts - especially where we need to determine unequivocally whether a condition is pathological-it seems to require an element of human decision. This itself does not need to be dubious, but rather normal procedure in relation to vague terms. In the philosophical debate, where to draw the line is normally regarded a problem for two distinguishable reasons: First, because it might involve an element of value judgement. This would threaten specifically the ambitions of a naturalist account of disease (Miller Brown 1985, 5f.; but cf. Veit 2021; Amoretti and Lalumera 
2021). Second, line drawing could be problematic because it might not allow for any answer that is reasonable, non-arbitrary and "workable" (Schwartz 2017, 495). I will mainly focus on the second interpretation of the line-drawing problem and suggest a scientific response. The first interpretation of the problem requires further considerations regarding what types of value judgements are involved when drawing the line between dysfunction and normal function. Although I cannot go into detail here, I believe that any evaluations that might be involved will not be based on individual or social value judgements (Schramme 2010), but refer to the natural normativity of biological functions; hence be grounded on a scientific explanation of abnormality (Matthewson and Griffiths 2017, 452).

Interestingly, some organismic functions are not affected by the quantitative problem, at least they might relatively easily allow for nonarbitrary and workable results. There are some effects that only allow for absolute levels of performance. For instance, a two-way switch is either fulfilling its function or dysfunctional, depending on whether it can be turned or not. There are similar kinds of mechanisms in human organisms where the threshold of dysfunction is straightforward, even if the function does allow for grades. A function of the ovaries is, for instance, to produce eggs. At least, this is a function of the ovaries during a particular period of the life of a female organism. If a token ovary does not produce eggs, it is dysfunctional in that respect. So, in cases such as the one just mentioned, the way to tackle the quantitative problem is straightforward.

My example seems to raise concerns, however, that lead us into less straightforward terrain: After all, isn't the function of the ovaries to produce fertile eggs, at the right time, as well as, probably, to only produce one egg within one cycle? Being fertile seems to clearly allow for gradual aspects, for instance regarding how likely an ovum is to develop into a zygote, once fertilised. How fertilisable an ovum is clearly depends on numerous other functions and environmental conditions. My example was mainly meant to establish the possibility of isolated functions, where thresholds are relatively easy to determine, not to exclude more complicated gradual functions. If ovaries do not produce eggs-never mind any gradable characteristics of these - then they are dysfunctional. If they produce more than one ovum during a cycle, they might also be dysfunctional. Whether this is the case or not does not matter for my purpose, as I will agree that additional, non-biological considerations are required for determining dysfunctions in a clinical context. 
The quantitative problem is more difficult to tackle when traits allow for different levels of performance without clear thresholds. The most common way to provide a threshold, at least in abstract terms, is to say that a trait is dysfunctional if it does not perform efficiently. In the philosophy of medicine, Boorse, who defines disease as impairment of functional ability, has described the threshold of dysfunction in the following way:

Normal functioning in a member of the reference class is the performance by each internal part of all its statistically typical functions with at least statistically typical efficiency, i.e. at efficiency levels within or above some chosen central region of their population distribution. (Boorse 1977, 558f.)

The important point here is to be found in the final part of the sentence. Boorse makes clear that he wants to account for the threshold by statistical means. There are, however, serious problems with such a framework (Schwartz 2007; see also Davies 2001, 186).

As mentioned before, several authors have objected that a statistical answer to the line-drawing problem is arbitrary. If this is true, it might, firstly, show that dysfunction cannot after all be explained in purely valueneutral, scientific terms. The quantitative problem might require reference to particular human interests, which Boorse would like to exclude from his theory of disease. Secondly, his theory leads to problems with low levels of functioning that are prevalent in a population. A statistical analysis does not work if inefficient levels are statistically normal. I will briefly deal with both of these objections, before presenting an alternative answer to the quantitative problem. I consider my considerations to apply within a generally Boorsean theoretical outlook. There might be alternative theories of function, for instance Cummins-style systemic theories of function (Cummins 1975) that fare better with the quantitative problem. The main purpose of my paper is, however, to present an alternative reply based on Boorse's framework.

Boorse himself maintains, in the quoted sentence, that the exact boundary between efficient function and dysfunction is "chosen", i.e. determined by human choice. However, he insists that the chosen region within the population distribution, which is deemed to be below the efficient level of functioning, is not chosen for reasons of human welfare interests, or the like, but for reasons of statistical theory. He says that 'deficiency', according to his account, is an "arithmetic, not an evaluative, concept" (Boorse 1997, 21). This might be so, but it nevertheless introduces an element of human decision about where to draw the line of pathological 
levels of functioning. Indeed, Boorse himself says that "the lower limit of normal functional ability - the line between normal and pathological - is arbitrary" (Boorse 1987, 371).

Here the second problem looms, as there are some low levels of performance, which are so common that they will never stick out statistically. A common way to deal with this problem has been to put the relevant functions in relation to normal environments (Hausman 2014). Boorse addressed the related problem of statistically common or universal diseases, such as caries, already in his early papers. He also laid out a theory that refers to an environmental clause, so that environmentally caused or sustained dysfunctions are not deemed diseases (Boorse 1977, 566ff.). All these fixes seem to lead to the conclusion that normal levels of functioning cannot wholly be determined intrinsically, that is, only by reference to the organism and its internal mechanisms itself. This might not be devastating, but nevertheless, to include normal environments in the definition of normal functioning simply shifts the problem as to where the threshold of abnormality lies from one aspect to a perhaps even more contested one (see also Kingma 2010).

It is hard to deny that the exact level of performance needed in order to fall within the area of normal functioning is difficult to draw and indeed vague. The reference to statistics makes this even more evident. After all, there are no logical or conceptual reasons to see any normative significance in, say, the fact of two standard deviations in any measured value. Boorse says: "whenever one knows the goal of a process, one knows what is more or less function, and 'deficiency', in the context quoted, simply means much less than average" (Boorse 1997, 21). For Boorse, "functional efficiency [is] measurable" (Boorse 2014, 690; see also Kraemer 2013) and the boundary to dysfunction is due to a significant distance from the statistically determined mean level of functioning. Yet to concede that there is necessarily an element of human choice involved actually underlines the point which critics have brought forward. Critics say that this feature, the element of human choice, challenges Boorse's claim of providing a value-free theory of disease (Schwartz 2007; Kingma 2010).

However, I believe Boorse's reliance on statistics is wrongly conceived. Statistics is only an instrument to gain knowledge about organisms, not itself the source of drawing the line between function and dysfunction. It is important to see that the ontological perspective on the boundary between function and dysfunction is different from an epistemological perspective. The ontological perspective has to do with the level of performance of a type of trait; the epistemological perspective is required 
to gain knowledge about the required level of functioning (cf. Hauswald and Keuck 2017).

The fact that we are here referring to types should lead us to acknowledge that setting the ontological boundaries requires a certain amount of abstraction and idealization. Surely the level of normal performance of a mechanism is not straightforward. Still, in cases of organisms that are structured through different levels of sub-systems the thresholds are determined by the relevant effect that is minimally required to maintain the relevant subsystem altogether. This is mainly, although probably not exclusively (because some environmental factors might need to be acknowledged), due to characteristics of the type of organism itself.

The quantitative level of normal performance for an organismic mechanism or process is determined by the requirement of achieving the effect that is its function. In other words, we need to see the performance of a trait as a means to an end (McLaughlin 2009, 96ff.). The end of a functional mechanism is a particular effect. Any level that achieves the effect is normal; any level of performance - high or low - that fails to maintain or to lead to the required effect is dysfunctional. Hence, the threshold of functional efficiency is determined by specific effects of biological processes.

So far, my argument seems to be circular: The line identifying the level of efficient functioning is drawn by the function of a trait. A trait is functional if it fulfils its function; dysfunctional if it does not fulfil its function. However, the required effects are themselves to be seen in relation to the hierarchical organisation of organisms. An effect is needed, usually together with other effects, to maintain functioning on a more complex level. Hence, effects (i.e. functions) of a trait are means to other ends. For instance, a function such as hearing requires many sub-functions being achieved. A heart needs to fulfil its functions to maintain other systems in the organism. It is not arbitrary or unworkable to determine the amount of blood pumping to achieve these other effects.

It needs to be stressed again that my suggested response to the quantitative problem is still not a solution to the problem as such, in the sense of getting rid of it once and for all. I nevertheless hope to show that this is not damaging, because at least my response opens a way of identifying reasonable, non-arbitrary and workable thresholds. It is true that the effects (functions) of connected organismic systems, which are supposed to determine the level of functions in maintaining traits, are themselves usually gradable. Hence the quantitative problem does not dissolve. For 
instance, the threshold of cardiac output-say, 5 litres per minute-is effective in relation to a gradable performance of the organism. Now, we need to know what level of organismic performance we are using as baseline. The relevant effect might be required in a state of rest, whilst running, or in any other possible conditions of an organism. Yet, once we have settled on the respective effects of an overall system, due to our research interest, what level of functioning is required for maintaining the systemic functions is a matter of fact.

Biology can account for several functional systems within a type of organism and for their interdependence (Saborido et al. 2016). At least in biological theory, the ontological perspective on drawing the quantitative boundary between normal levels of performance and dysfunction-even where there are grades of performance - can be addressed by purely factual considerations. After all, the exact level of required or normal performance is determined by the factual question as to whether a particular effect can (still) be achieved. It should be added that this also allows for compensatory mechanisms to take over a function or making up for quantitative loss (Saborido et al. 2016, 113).

Boorse himself had stated a similar idea in an early essay:

In fact, the structure of organisms shows a means-end hierarchy with goal-directedness at every level. Individual cells are goaldirected to manufacturing certain compounds; by doing so they contribute to higher-level goals like muscle contraction; these goals contribute to overt behavior like web-spinning, nestbuilding, or prey-catching; overt behavior contributes to such goals as individual and species survival and reproduction. What I suggest is that the function of any part or process, for the biologist, is its ultimate contribution to certain goals at the apex of the hierarchy. (Boorse 1977, 556)

I do not believe that we need to endorse Boorse's idea of a hierarchy of functions including an apex. In other words, we do not need to assume that biological systems have overall purposes, such as survival or reproduction in case of Boorse's theory. This assumption has raised numerous concerns (Cooper 2002). It is sufficient to agree with the interpretation of organisms as a conglomerate of subsystems involving functions on different levelswhere levels is meant as a spatial term.

The way I have interpreted the quantitative problem makes clear that the relevant concept is not, as Boorse has claimed, functional efficiency, but 
rather functional effectivity. In contrast to efficiency, the notion of effectivity comes with an internal absolute threshold, namely whether a specific effect is reached or not. In relation to the threshold a level of performance is either effective or not. In this reading there is no grey area. This shows, to my mind, that the suggested answer to the quantitative problem is in congruence with the straightforward cases of complete failure of any level of function. After all, not reaching the effect, which is the function of a trait, is simply failure of relevant performance.

It is true, of course, that we can introduce gradual interpretations of functioning, for instance regarding hearing. A person might be able to hear better or worse. I am suggesting, though, that once we will have determined an ideal type of the functional system for human hearing, we can decide whether the person's hearing is dysfunctional without considering its comparative level of overall performance. Normal hearing will be understood as a set of functions performing effectively on different interlinking levels. These functions will, at least for eventual clinical purposes, need to be modelled relative to age in order to produce reasonable thresholds that take senescence into account. To be sure, some of these functions will be set by quantitative measures, but the quantitative threshold levels will be determined by the respective required effects to maintain a system of functions. In other words, the thresholds of quantitative functioning will be set by the necessities of maintaining an organismic system. These are determined by an idealised model of a type of organism, relative to certain additional features, such as age or sex. Such an idealised model is the product of humans, of course. But it is not based on unreasonable, arbitrary or unworkable assumptions.

The ontological perspective, I have said earlier, is different from the epistemological perspective. Indeed, it is obvious that it is not easy at all in practice to establish the exact boundaries of normal function and dysfunction, though we have a theoretical instrument in modelling an ideal type of a functioning organism. I believe this is where statistical considerations can be of some importance (see also Hausman 2014; Garson and Piccinini 2014, 10ff.). After all, we cannot simply read quantitative values of normal performance off nature, but need to determine them by studying real specimens of the relevant organisms. Hence, we might use statistics as a means to gain knowledge about these levels of normal functioning. Yet we should now be able to see that statistics only provides clues for supporting certain theoretical assumptions about the ontological threshold between normal functioning and dysfunction. Statistics cannot itself establish the ontological boundaries, because the latter are determined by biological facts. We have seen already that statistics might 
also lead to epistemic problems in cases of endemic malfunction or universal diseases. However, statistics is not our only means of gaining knowledge about functions. In biology, reverse engineering, for instance, is a common mode of developing models of the functioning of organisms (Smith 1995, 3; Green 2018).

\section{Biological and Clinical Dysfunction}

So far, I have discussed the quantitative problem as a classificatory issue within biology. And it is such a problem, of course. We want to know where to draw the boundaries between normal function and dysfunction, and this task need to be performed in relation to the organisms we study. Hence, we focus on the biological features of a specific type of organism to establish a prototype. But the quantitative problem in medicine is not only a biological issue; it is a clinical issue as well. We need additional considerations in this perspective.

I started by pointing out the normative significance of the threshold between normal function and dysfunction for calling a condition a disease. Most importantly, the boundary has an impact on people's access to publicly funded healthcare resources. Medical classification relies on a theory of the abnormal functioning of a specific type of organism, so the clinical perspective builds on biological considerations. A medical nosology for human beings, for instance the International Classification of Diseases (ICD), builds on a kind of normative prototype of a healthy human being - or rather it gathers several prototypes of abnormal functioning, specified according to different systems of organismic organisation. The ICD is organised along diseases of the blood, of the immune system, of metabolism, the nervous system, the visual system, and so on. But classification for clinical purposes does not stop at biological considerations. Clinical prototypes already contain pragmatic elements, which have to do with non-biological aspects, such as whether a condition can be identified or treated by medical means and has any impact on human wellbeing (see Cooper 2020, 154f.).

To be sure, we can imagine a medical nosology that rests exclusively on a biological foundation. Medical terminology indeed contains the term "subclinical", which might at least partially account for a purely biological perspective. I assume that the notion of the subclinical is actually intended to record early stages of processes that might (very likely) result in disease, though are not themselves instances of disease. Similarly, a purely biological classification would serve the purpose of recording any known 
organismic dysfunction. This might be a relevant purpose for medicine as a scientific endeavour. But surely the purpose of such a system would be purely biological, not clinical. Indeed, to merge the biological and the clinical perspective easily lends itself to the problem of overdiagnosis. There are many biological dysfunctions that will not have serious effects on overall organismic functioning in any token organism, especially at a more microscopic level of the functional system. To call all biological dysfunctions diseases can have serious practical consequences because of the normative effects that usually come with the use of a disease label.

In this context, it has been said that Boorse's theory of disease is overly inclusive, because it rests on dysfunction, and dysfunction can surely be present on a cellular level. Hence even "one dead cell" would be pathological, according to Boorse's theory, which seems counterintuitive (Nordenfelt 1995, 28; Wakefield 2014, 656; Doust et al. 2017). Boorse himself has responded to this objection by accepting the implication and maintaining that every person has at any time some pathological condition, if only very minor, of course (Boorse 1997, 50f., 85; see also Boorse 2014, 706; 2015). But I believe we can respond to the charge of overinclusiveness by pointing out that the classification of dysfunctions for clinical purposes, i.e. the classification of diseases, is different from a purely biological classification of dysfunction. It might be true that everyone has at any given time a biological dysfunction present in their organism. But the concept of dysfunction for clinical purposes adds further criteria to eventually result in the concept of disease.

I doubt that these additional criteria are convincingly understood by simply adding a harm condition, as some authors, most notably Jerry Wakefield, would like to convince us (Wakefield 1992; 2014). Clinical classification serves several aims, which I cannot thoroughly discuss in this paper (for an interesting analysis from a historical perspective, see Jutel 2011). There seem to be numerous examples of clinical diseases (listed in the ICD-11) that are not themselves harmful, say, for instance, benign skin tags (code EK 71.0) or protruding ears (code LA 21.1.). These conditions usually are considered for medical treatment, that is, they in fact qualify as entry ticket to use publicly funded resources. For the present purposes, however, my objection to an added harm criterion is not particularly relevant. It is more important to point out that although biological considerations build the basis of medical nosology, biological dysfunction is not sufficient for disease from a clinical perspective. We need a clinical understanding of dysfunction as well. One dead cell will not be seen as pathological from a clinical perspective. 
I should stress that these additional criteria for clinical purposes bear on the quantitative problem of drawing the boundary between normal function and dysfunction. Although biological dysfunction is based on factual aspects regarding traits not achieving their supposed effects, the concept of clinical dysfunction is not merely based on factual aspects. Still, I want to argue that the additional considerations for clinical purposes do not undermine the foundational factual elements of biological dysfunction.

An example that has been discussed to show that Boorse's account has problems with drawing the line between disease and health is hypertension (Rogers and Walker 2017, 410). The exact line of a pathologically high blood pressure seems arbitrary, in other words not factual at all. How would this example pan out in the account I have introduced? It would need to be checked what quantitative value of blood pressure, if any, typically goes along with a lack of achieving the effects of related functions of the vascular system. As I have said earlier, we would need to abstract from individual cases and devise a normative prototype of normal blood pressure. Now, the specific example might appear not be pertinent, anyway, because it seems that blood pressure itself is not a functional feature of organisms, but merely a symptom of possible dysfunctions, especially of future dysfunctions (see Hofmann 2021, 131). Still, quantitative levels of blood pressure are indications of levels of functioning. Very high values of blood pressure are causally associated with pathological conditions, especially heart and kidney diseases. To be sure, this is a statistical correlation, indicating a specific risk of disease, not disease itself. In some cases, abnormal blood pressure might be a sign of a dysfunction, but again hypertension itself would not constitute dysfunction. Altogether, blood pressure is not a straightforward example of a functional trait. It is not clear whether it poses specific problems for a scientific theory of disease, because the quantitative threshold would be set by the requirements of maintaining the relevant organismic system.

Additionally, within the abnormal range, we might want to further enquire, from a clinical perspective, whether all subnormal levels are posing risks for human wellbeing or affect any other additional criteria. Still, these additional considerations would only be pursued below the threshold set by biological considerations. In other words, only biological dysfunctions would qualify as clinical dysfunctions. Hence there is no special danger of including too many conditions as diseases, in other words, no concern of pathologisation or overdiagnosis.

To be sure, I have only discussed one example that was used in the scholarly literature to establish the arbitrary nature of attempts to tackle the 
quantitative problem. There might be other, more pertinent cases, which could undermine my claim that biological dysfunction sets the boundaries for determining clinical dysfunction. But as long as these can be accommodated, my claim regarding the scientific boundaries of clinical dysfunction still stands.

In summary, I have argued that the quantitative threshold lies where the specific effect, which is a trait's function, cannot be achieved or maintained. This relates to the biological notion of dysfunction. In a clinical context, there will be additional considerations. Still, these need to be based on the biological account. There can only be pathology where there is biological dysfunction (Matthewson and Griffiths 2017, 449; cf. Hucklenbroich 2017). Not every biological dysfunction is necessarily a case of clinical dysfunction, as we have seen when briefly discussing the "one dead cell" problem.

It is easily imaginable that we will have different quantitative measures for clinical purposes, which are more lenient, as it were. For instance, any value of myopia might be dysfunctional from a biological point of view, at least if we disregard aspects of normal deterioration of eyesight due to senescence for the time being. After all, the very notion of myopia seems to be based on an assessment of a trait as dysfunctional. The effect of sharp representation of an image on the retina is not achieved if an organism has myopia. However, clinically speaking it is likely that we will accept minor levels of myopia within the normative prototype, perhaps because perfect eyesight is so rare or because it normally does not bother people. Accordingly, there are external values and human interests involved when drawing the boundary to those biological dysfunctions that are clinically pathological.

Similarly, in psychiatry it is common to include in the classification of several disorders a clause that a specific condition must be present for more than six months. From a biological point of view, if a mental dysfunction is present, it will be present at any point in time, not just after some period of time. To be sure, we might use the time factor for epistemic reasons, in order to gain sufficient knowledge about the actual mechanism and whether it is still functioning in an individual person. But be that as it may, the biological and the clinical perspective can fall apart, simply because the relevant thresholds can differ (see Cooper 2013).

The fact that clinical considerations are partly driven by human interests, mainly by considerations of the impact of a biological dysfunction on wellbeing or the possibility of treatment, should not conceal the other fact 
that within this perspective the concept of disease still has a firm scientific basis in biological dysfunction. Only biological dysfunctions can be deemed diseases, though not all will. This is different from accounts of disease that start from a social-evaluative foundation. The account developed here helps avoiding pathologisation of normal conditions and can be instrumental in preventing overdiagnosis. Admittedly, the latter achievement depends on the characteristics of non-scientific elements used in actual medical practice. It is true that in many countries medicine tends to cater for ever more minor biological dysfunctions and even for other conditions that are not biological dysfunctions at all. But this problem is a political one and scientific theories of disease cannot be blamed for it.

\section{The Role of Diagnosis}

So far, I have discussed the quantitative problem in relation to what I have called normative prototypes, hence on a generic level. It is a problem for medical nosology. But assessments regarding dysfunction in clinical medicine are also made on an individual level. Doctors make statements about individual tokens of organisms, also known as patients. These medical judgements are called diagnoses. The process of medical diagnosis leads to further complications for the quantitative problem of the boundary between normal functioning and dysfunction, because it opens some space for individual deviation from a normative prototype. The specific situation of a patient, who is of course not merely regarded as an organism when presented to a doctor, partly drives the assessment of functional capacity. A condition that is clearly clinically dysfunctional and hence pathological according to the relevant classification might not be diagnosed thus by a doctor. It might happen that an individual will not be subsumed under a prototype despite fulfilling the criteria of inclusion.

In terms of the quantitative problem the flexibility for diagnosis might work both ways; that is, there might be a diagnosis of a pathological condition where the individual patient is within the area of clinically normal functioning. For instance, for professional sharpshooters even the slightest level of myopia might have devastating effects on their career. Accordingly, a doctor might diagnose a relevant pathology. Note that this is different from diagnosing an alleged disease outside the range of biological dysfunction. A sharpshooter might prefer to have a vision comparable to an eagle; but biologically normal levels of human functioning can never be diseases within the suggested theory. 
There might also be reasons for a doctor to avoid diagnosing a disease although the person presents with a clinically abnormal value. For instance, a teenager with extremely tall parents might have a growth hormone dysfunction, leading to stunted growth, but also resulting in a predicted height that is statistically normal. In such a case, it does not seem required to diagnose a disease. Such scope for deviance from clinical classification is actually desirable, because clinical classification by its very nature cannot account for individual cases. Yet, in medical practice it is important to do justice to individual cases.

It should also be stressed that any judgement regarding disease in individual cases is due to a diagnostic process. Perhaps in contrast to common expectation, the presence of disease is never fully established by pathological findings alone-which might for instance be achieved by investigating samples of tissue. Diagnoses are made by specialist doctors in relation to a patient. Their verdict is of course informed by pathologists' reports, but an individual judgement regarding disease within the clinical context is not merely due to a finding of clinical dysfunction. Admittedly, it seems that this practice is changing in reality and doctors tend to look more at laboratory results than at the patient to draw a diagnosis. But this development actually undermines the significant difference between the biological and the clinical perspective and should therefore be criticised.

If medical nosology could always sufficiently determine whether an individual case falls under a type of disease, the exercise of diagnosis would be merely deductive. Potentially a computer could then do the diagnosis, because the only question would be whether a person, or case for that matter, presents certain conditions, which form the criteria of a specific concept of disease, defined in a classificatory system, such as the ICD. But diagnosis is not simply a deductive exercise, and it should not be. Surely this aspect of the quantitative problem involves evaluative considerations that transcend the mainly scientific or factual aspects I have discussed above (cf. Whitbeck 1981).

\section{Conclusion}

In this paper, I have defended a particular way of accounting for the boundary between normal biological functioning and dysfunction. I claim that this boundary is due to matters of fact, yet not constituted by statistical realities. The quantitative problem can be dealt with in a non-arbitrary way. Functions are specific effects, which are either achieved or not. This is a factual question about the quantitative necessities to perform biological 
functions within a complex structured organism. In virtue of exploring the systems of organismic functioning, biology develops normative prototypes, which can be used for medical purposes. However, when switching to a clinical perspective, additional considerations are introduced. Hence biological dysfunction is not the same as disease. Matters become even more complicated with individual diagnoses, which establish the reality of instances of disease in the actual practice of medicine. Medical diagnosis requires a judgement that puts clinical types and individual persons in relation.

\section{Acknowledgments}

I would like to thank Dan Hausman, Ulrich Krohs, Steve McLeod, Wendy Rogers, Peter Schwartz and two anonymous reviewers for their helpful comments.

\section{REFERENCES}

Amoretti, M. Cristina, and Elisabetta Lalumera. 2021. "Wherein Is the Concept of Disease Normative? From Weak Normativity to Value-Conscious Naturalism." Medicine, Health Care and Philosophy, August. https://doi.org/10.1007/s11019-021-10048$\mathrm{x}$.

Ariew, André, Robert Cummins, and Mark Perlman. 2002. Functions: New Essays in the Philosophy of Psychology and Biology. Oxford University Press.

Boorse, Christopher. 1977. "Health as a Theoretical Concept." Philosophy of Science 44 (4): 542-73. https://doi.org/10.1086/288768.

-. 1987. "Concepts of Health." In Health Care Ethics: An Introduction, edited by Donald Van De Veer and Tom Regan, 359-93. Temple University Press.

1997. "A Rebuttal on Health." In What Is Disease?, edited by James M. Humber and Robert F. Almeder, 3-134. Biomedical Ethics Reviews. Totowa, NJ: Humana Press. https://doi.org/10.1007/978-1-59259-451-1_1. 2014. "A Second Rebuttal on Health." Journal of Medicine and Philosophy 39 (6): 683-724.

. 2015. "Reply to Wakefield on Harm and Health." MS.

Brown, Miller W. 1985. “On Defining 'Disease'.” Journal of Medicine and Philosophy 10 (4): 311-28. https://doi.org/10.1093/jmp/10.4.311. 
Cooper, I-Rachel. 2020. "The Concept of Disorder Revisited: Robustly Value-Laden Despite Change." Aristotelian Society Supplementary Volume 94 (1): 141-61. https://doi.org/10.1093/arisup/akaa010.

Cooper, Rachel. 2002. "Disease." Studies in History and Philosophy of Science Part C: Studies in History and Philosophy of Biological and Biomedical Sciences 33 (2): 263-82. https://doi.org/10.1016/S0039-3681(02)00018-3. 2013. "Avoiding False Positives: Zones of Rarity, the Threshold Problem, and the DSM Clinical Significance Criterion." The Canadian Journal of Psychiatry 58 (11): 606-11. https://doi.org/10.1177/070674371305801105.

Cummins, Robert. 1975. "Functional Analysis.” Journal of Philosophy 72, 741-65.

Davies, Paul Sheldon. 2001. Norms of Nature: Naturalism and the Nature of Functions. A Bradford Book. Cambridge, Massachusetts: MIT Press.

Doust, Jenny, Mary Jean Walker, and Wendy A. Rogers. 2017. "Current Dilemmas in Defining the Boundaries of Disease." The Journal of Medicine and Philosophy: A Forum for Bioethics and Philosophy of Medicine 42 (4): 350-66. https://doi.org/10.1093/jmp/jhx009.

Engelhardt, H. T. 1976. "Ideology and Etiology." Journal of Medicine and Philosophy 1 (3): 256-68. https://doi.org/10.1093/jmp/1.3.256.

Garson, Justin. 2016. A Critical Overview of Biological Functions. 2019. What Biological Functions Are and Why They Matter. Cambridge: Cambridge University Press. https://doi.org/10.1017/9781108560764.

Garson, Justin, and Gualtiero Piccinini. 2014. "Functions Must Be Performed at Appropriate Rates in Appropriate Situations." The British Journal for the Philosophy of Science 65 (1): 1-20. https://doi.org/10.1093/bjps/axs041.

Godfrey-Smith, Peter. 1993. "Functions: Consensus without Unity." Pacific Philosophical Quarterly 74 (3): 196-208.

https://doi.org/10.1111/j.1468-0114.1993.tb00358.x.

Goosens, William K. 1980. "Values, Health, and Medicine." Philosophy of Science 47 (1): 100-115. https://doi.org/10.1086/288912.

Green, Sara. 2018. "Philosophy of Systems and Synthetic Biology." The Stanford Encyclopedia of Philosophy (Summer 2018 Edition), Edward N. Zalta (Ed.). 2018.

$<$ https://plato.stanford.edu/archives/sum2018/entries/systemssynthetic-biology/>. 
Griffiths, Paul E., and John Matthewson. 2016. "Evolution, Dysfunction, and Disease: A Reappraisal." The British Journal for the Philosophy of Science 69 (2): 301-27. https://doi.org/10.1093/bjps/axw021.

Hausman, D. M. 2014. "Health and Functional Efficiency." Journal of Medicine and Philosophy 39 (6): 634-47. https://doi.org/10.1093/jmp/jhu036.

Hauswald, Rico, and Lara Keuc. 2017. "Indeterminacy in Medical Classification: On Continuity, Uncertainty, and Vagueness." In Vagueness in Psychiatry, edited by Geert Keil, Lara Keuck, and Rico Hauswald, 93-117. Oxford: Oxford University Press.

Hofmann, Bjørn. 2021. "How to Draw the Line between Health and Disease? Start with Suffering." Health Care Analysis 29 (2): 12743. https://doi.org/10.1007/s10728-021-00434-0.

Hucklenbroich, Peter. 2017. "Disease Entities and the Borderline between Health and Disease: Where Is the Place of Gradations?" In Vagueness in Psychiatry, edited by Geert Keil, Lara Keuck, and Rico Hauswald, 75-92. Oxford: Oxford University Press.

Jutel, Annemarie. 2011. "Classification, Disease, and Diagnosis." Perspectives in Biology and Medicine 54 (2): 189-205.

https://doi.org/10.1353/pbm.2011.0015.

Kingma, Elselijn. 2010. "Paracetamol, Poison, and Polio: Why Boorse's Account of Function Fails to Distinguish Health and Disease." The British Journal for the Philosophy of Science 61 (2): 241-64. https://doi.org/10.1093/bjps/axp034.

Kraemer, Daniel M. 2013. "Statistical Theories of Functions and the Problem of Epidemic Disease." Biology \& Philosophy 28 (3): 423-38. https://doi.org/10.1007/s10539-013-9365-3.

Lemoine, Maël. 2013. "Defining Disease beyond Conceptual Analysis: An Analysis of Conceptual Analysis in Philosophy of Medicine." Theoretical Medicine and Bioethics 34 (4): 309-25.

https://doi.org/10.1007/s11017-013-9261-5.

Matthewson, John. 2020. "Does Proper Function Come in Degrees?"

Biology \& Philosophy 35 (4): 39. https://doi.org/10.1007/s10539020-09758-y.

Matthewson, John, and Paul E. Griffiths. 2017. "Biological Criteria of Disease: Four Ways of Going Wrong." The Journal of Medicine and Philosophy 42 (4): 447-66.

https://doi.org/10.1093/jmp/jhx004.

McLaughlin, Peter. 2009. "Functions and Norms." In Functions in Biological and Artificial Worlds: Comparative Philosophical Perspectives, edited by Ulrich Krohs and Peter Kroes, Springer, 93-102. 
Melander, Peter. 1997. "Analyzing Functions: An Essay on a Fundamental Notion in Biology." Acta Universitatis Umensis 138. Stockholm: Almqvist \& Wiksell International.

Millikan, Ruth Garrett. 1989. "In Defense of Proper Functions." Philosophy of Science $56 \quad$ (2): 288-302. https://doi.org/10.1086/289488.

Neander, Karen. 1991. "The Teleological Notion of "Function"." Australasian Journal of Philosophy 69 (4): 454-68. https://doi.org/10.1080/00048409112344881.

1995. "Misrepresenting \& Malfunctioning." Philosophical Studies 79 (2): 109-41. https://doi.org/10.1007/BF00989706.

Nordenfelt, Lennart. 1995. On the Nature of Health: An Action-Theoretic Approach. Dordrecht: Springer.

Rogers, Wendy A., and Mary Jean Walker. 2017. "The Line-Drawing Problem in Disease Definition." The Journal of Medicine and Philosophy 42 (4): 405-23. https://doi.org/10.1093/jmp/jhx010.

Saborido, Cristian, Alvaro Moreno, María González-Moreno, and Juan Carlos Hernández Clemente. 2016. "Organizational Malfunctions and the Notions of Health and Disease." In Naturalism in the Philosophy of Health, edited by Élodie Giroux, 101-20. Cham: Springer. https://doi.org/10.1007/978-3-319-29091-1_7.

Schramme, Thomas. 2010. "Can We Define Mental Disorder by Using the Criterion of Mental Dysfunction?" Theoretical Medicine and Bioethics 31 (1): 35-47. https://doi.org/10.1007/s11017-0109136-y.

2019. Theories of Health Justice: Just Enough Health. London New York: Rowman \& Littlefield International.

Schroeder, S. Andrew. 2013. "Rethinking Health: Healthy or Healthier Than?" The British Journal for the Philosophy of Science 64 (1): 131-59. https://doi.org/10.1093/bjps/axs006.

Schwartz, Peter H. 2007. "Defining Dysfunction: Natural Selection, Design, and Drawing a Line*." Philosophy of Science 74 (3): 364 85. https://doi.org/10.1086/521970.

2017. "Progress in Defining Disease: Improved Approaches and Increased Impact." The Journal of Medicine and Philosophy 42 (4): 485-502. https://doi.org/10.1093/jmp/jhx012.

Smith, John Maynard. 1995. “Genes, Memes, \& Minds.” New York Review of Books, 1995.

https://www.nybooks.com/articles/1995/11/30/genes-memesminds/.

Tresker, Steven. 2020. "Theoretical and Clinical Disease and the Biostatistical Theory." Studies in History and Philosophy of 
Science Part C: Studies in History and Philosophy of Biological and Biomedical Sciences 82: 101249. https://doi.org/10.1016/j.shpsc.2019.101249.

Varga, Somogy. 2020. "Epistemic Authority, Philosophical Explication, and the Bio-Statistical Theory of Disease." Erkenntnis 85 (4): 937-56. https://doi.org/10.1007/s10670-018-0058-9.

Veit, Walter. 2021. "Biological Normativity: A New Hope for Naturalism?" Medicine, Health Care and Philosophy 24 (2): 291301. https://doi.org/10.1007/s11019-020-09993-w.

Wakefield, J. C. 2014. "The Biostatistical Theory Versus the Harmful Dysfunction Analysis, Part 1: Is Part-Dysfunction a Sufficient Condition for Medical Disorder?" Journal of Medicine and Philosophy 39 (6): 648-82. https://doi.org/10.1093/jmp/jhu038.

Wakefield, Jerome C. 1992. "The Concept of Mental Disorder: On the Boundary between Biological Facts and Social Values." American Psychologist 47 (3): 373-88. https://doi.org/10.1037/0003-066X.47.3.373.

Whitbeck, Caroline. 1981. "What Is Diagnosis? Some Critical Reflections." Metamedicine 2 (3): 319-29.

https://doi.org/10.1007/BF00882078.

Wouters, Arno G. 2003. "Four Notions of Biological Function." Studies in History and Philosophy of Science Part C: Studies in History and Philosophy of Biological and Biomedical Sciences 34 (4): 633-68. https://doi.org/10.1016/j.shpsc.2003.09.006.

Wright, Larry. 1973. "Functions." The Philosophical Review 82 (2), 13968. 
